\title{
Zircon to scheelite phase transition induced by pressure and magnetism in $\mathrm{TbCrO}_{4}$
}

\author{
E. Climent Pascual, * J. M. Gallardo Amores, and R. Sáez Puche \\ Departamento de Química Inorgánica I, Facultad de Ciencias Químicas, Universidad Complutense de Madrid, 28040 Madrid, Spain
}

M. Castro

Instituto de Ciencia de Materiales de Aragón, CSIC, Universidad de Zaragoza, 50009 Zaragoza, Spain

N. Taira

Chemistry Department, Gunma College of Technology, 580 Toribamachi, Maebashi, Gunma 371-8530, Japan

J. Romero de Paz

CAI Técnicas Físicas, Facultad de Ciencias Físicas, Universidad Complutense de Madrid, E-28040 Madrid, Spain

L. C. Chapon

ISIS Facility, Rutherford Appleton Laboratory-CCLRC, Chilton, Didcot, Oxfordshire OX11 OQX, United Kingdom

(Received 27 January 2009; revised manuscript received 10 March 2010; published 21 May 2010)

\begin{abstract}
The scheelite form of the $\mathrm{TbCrO}_{4}$ oxide has been obtained by treating $\mathrm{TbCrO}_{4}$-zircon at $4 \mathrm{GPa}$ and $823 \mathrm{~K}$. $\mathrm{X}$-ray and neutron diffraction data reveal that the high-pressure polymorph of $\mathrm{TbCrO}_{4}$ crystallizes with tetragonal symmetry space group $I 4_{1} / a$ and lattice parameters $a=5.03674(10) \AA$ and $c=11.3734(4) \AA$. Although bisdisphenoids $\left[\mathrm{TbO}_{8}\right]$ and tetrahedra $\left[\mathrm{CrO}_{4}\right]$ are present in both scheelite and zircon polymorphs, the remarkable changes observed in both polyhedra appear to support the reconstructive model for the zircon-scheelite first-order phase transition. Specific heat and neutron diffraction measurements confirm the antiferromagnetic ordering previously proposed from magnetic susceptibility measurements, in which both $\mathrm{Tb}^{3+}$ and $\mathrm{Cr}^{5+} \mathrm{sub}^{-}$ lattices are involved. The magnetic structure has been determined and can be described on the basis of the coincidence between the chemical and magnetic cells, $\mathbf{k}=(0,0,0)$, where the magnetic moments of the $\mathrm{Tb}^{3+}$ and $\mathrm{Cr}^{5+}$ cations are antiferromagnetically aligned along the $c$ axis of the structure, with $T_{N}=29 \mathrm{~K}$. Their values at $2 \mathrm{~K}$ are $8.97(3) \mu_{B}$ and $1.089(9) \mu_{B}$, respectively.
\end{abstract}

DOI: 10.1103/PhysRevB.81.174419

PACS number(s): 75.25.-j, 62.50.-p, 61.66.Fn, 75.30.Cr

\section{INTRODUCTION}

Rare earth chromates $\mathrm{RCrO}_{4}$ belong to the family of compounds of general formula $R X \mathrm{O}_{4}$, where $R$ is a rare earth element and $X=\mathrm{P}, \mathrm{As}, \mathrm{Cr}, \mathrm{V},{ }^{1,2}$ which crystallize in two structural types depending on the sizes of the rare earth trivalent cation and the $X$ element. ${ }^{3}$ In the case of the $R \mathrm{CrO}_{4}$ oxides, the first member of this family of compounds $\mathrm{LaCrO}_{4}$ presents the monazite-type structure showing monoclinic symmetry space group (SG) $P 2_{1} / n$ (No. 14), ${ }^{4}$ while the $\mathrm{PrCrO}_{4}$ is dimorphic using specific synthesis conditions the zircon form has been successfully obtained as pure phase. ${ }^{5}$ The remaining members crystallize with zircon-type structure, showing tetragonal symmetry SG I4 $/$ amd (No. 141). ${ }^{6,7}$

Many studies have been reported concerning the crystallographic, magnetic, and optical properties of phosphates, arsenates, and vanadates..$^{8,9}$ By contrast the $R \mathrm{CrO}_{4}$ oxides have been much less studied because it is rather difficult to prepare them as pure phases, mainly due to the difficult to achieve the stabilization of the unusual $\mathrm{Cr}^{5+}$ oxidation state ${ }^{10,11}$ and the high tendency to be reduced to $\mathrm{Cr}^{3+}$ giving the very stable $\mathrm{RCrO}_{3}$ distorted perovskite phases.

The observation of pressure-driven phase transition in $R X \mathrm{O}_{4}$ compounds dates back to the early 1960s. Stubican et $a l .{ }^{12}$ reported that zircon or monazite structures of vanadates and arsenates transform to scheelite structure between 2 and $8 \mathrm{GPa}$. In the late $1980 \mathrm{~s}$ Bastide $^{13}$ tried to give a systematic explanation of the pressure induced phase transition for different families of $A B X_{4}$ compounds, but it has been only in the past decade that detailed studies of the high-pressure phases with scheelite-type structure have been done. ${ }^{14,15}$ Very recently, Errandonea and Manjon ${ }^{16}$ reviewed the studies at high pressures and temperatures of $A B X_{4}$ compounds in order to better understand the effect of pressure on the structure and physical properties. For the rare earth orthophosphates $R \mathrm{PO}_{4}$ pressure induced zircon to scheelite phase transitions in $\mathrm{YbPO}_{4}$ and $\mathrm{LuPO}_{4}$ have been reported. ${ }^{17}$ Concerning the chromates $\mathrm{RCrO}_{4}$ the zircon to scheelite phase transitions induced by pressure have only been studied for $\mathrm{YCrO}_{4},{ }^{18,19} \mathrm{HoCrO}_{4},{ }^{20}$ and $\mathrm{TbCrO}_{4} \cdot{ }^{21}$ Because this scheelite polymorph can be quenched to ambient conditions it will be possible to study its structural and physical properties. Accompanying this structural phase transition from zircon to scheelite the magnetic properties of these two polymorphs change dramatically. In this sense, we have recently observed that most of the zircon-type $R \mathrm{CrO}_{4}$ oxides are ferromagnetic, ${ }^{22}$ whereas their counterpart scheelite forms are antiferromagnetic. ${ }^{19-21}$

In this paper, we report the synthesis of the high-pressure scheelite polymorph of $\mathrm{TbCrO}_{4}$. The crystal structure, the magnetic structure and specific heat measurements are also reported, as well as structure-property relationships for the scheelite and zircon polymorphs in order to explain the different magnetic properties that they present. 


\section{EXPERIMENTAL DETAILS}

\section{A. Sample preparation}

Scheelite-polymorph $\mathrm{TbCrO}_{4}$ was prepared from a polycrystalline powder sample of zircon- $\mathrm{TbCrO}_{4}$, which was synthesized by heating in an oxygen flow using stoichiometric amounts of $\mathrm{Cr}\left(\mathrm{NO}_{3}\right)_{3} \cdot 9 \mathrm{H}_{2} \mathrm{O}$ and $\mathrm{Tb}\left(\mathrm{NO}_{3}\right)_{3} \cdot 6 \mathrm{H}_{2} \mathrm{O}$ according to the following thermal process: $30 \mathrm{~min}$ at $433 \mathrm{~K}, 30 \mathrm{~min}$ at $473 \mathrm{~K}$, and $12 \mathrm{~h}$ at $853 \mathrm{~K}$. This powdered zircon polymorph was packaged in a gold container and then inserted into a pyrophyllite assembly. A graphite tube was used as heater. A belt-type press was used to carry out the experiments in the 3-6 GPa pressure and 673-873 $\mathrm{K}$ temperature ranges. The selected conditions to obtain pure samples were $4 \mathrm{GPa}$ and $823 \mathrm{~K}$ for $30 \mathrm{~min}$; further increasing of pressure and temperature yields a large amount of $\mathrm{TbCrO}_{3}$ perovskite as impurity. The typical reaction path was as follows: (1) increasing the pressure up to $4 \mathrm{GPa}$; (2) rising the temperature up to $833 \mathrm{~K}$ in $15 \mathrm{~min}$; (3) maintaining there $30 \mathrm{~min}$; and (4) temperature quenching to room temperature. Afterwards, the pressure was slowly decreased to ambient pressure in $2 \mathrm{~h}$. The purity of the samples was tested by means of x-ray diffraction measurements.

\section{B. Structural analysis}

Powder neutron diffraction patterns to probe the crystal and magnetic structure were collected at low temperature using the high-resolution time-of-flight OSIRIS diffractometer at the ISIS Facility of the Rutherford Appleton Laboratory (U.K.). The sample was enclosed in a vanadium can placed in a helium cryostat. Long scans were recorded above and below the Néel temperature to solve the magnetic structure. Shorter scans in the $d$-spacing range $(3-5 \AA)$ were recorded between 2 and $40 \mathrm{~K}$ in steps of $1.5 \mathrm{~K}$ to follow the temperature dependence of the $(1 \overline{1} 0)(110)+k$ magnetic Bragg peak. Neutron diffraction data were analyzed with the Rietveld method using the FULLPROF program suite. ${ }^{23}$

\section{Specific heat measurements}

The specific heat data were taken with a quantum design physical property measurement system (PPMS). Measurements, based on the thermal relaxation method, ${ }^{24}$ were done in the temperature range of 5-300 $\mathrm{K}$ at applied magnetic fields from 0 to 9 T. A small portion of the sample $(\approx 4 \mathrm{mg}$ ) was fixed to the sapphire platform of the sample holder by a small amount of Apiezon grease. The specific heat of the sample holder and the grease was measured separately in the same conditions and this addendum was subtracted from the experimental values in order to obtain the specific heat of the scheelite-type oxides.

\section{RESULTS AND DISCUSSION}

\section{A. Structural characterization}

Figure 1(a) shows the observed, calculated and difference neutron diffraction profiles at $40 \mathrm{~K}$ for scheelite polymorph of $\mathrm{TbCrO}_{4}$. The structural parameters of scheelite $\mathrm{TbCrO}_{4}$

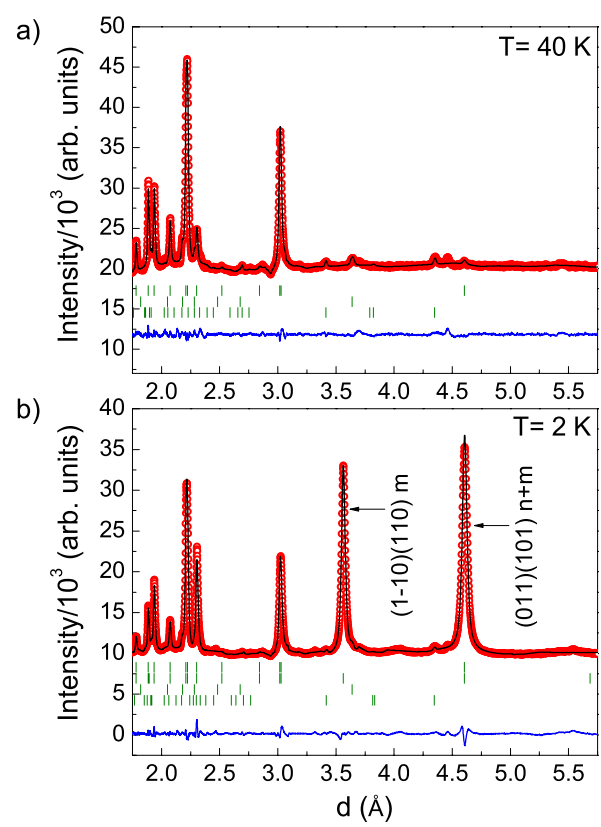

FIG. 1. (Color online) Rietveld refinement of neutron diffraction patterns obtained at (a) $40 \mathrm{~K}$ and (b) $2 \mathrm{~K}$ for scheelite-type $\mathrm{TbCrO}_{4}$. Observed, calculated and difference profiles are denoted by circles, the upper solid line and the lower solid line, respectively. Vertical marks denote the position of (a) nuclear allowed reflections for scheelite polymorph (first line), $\mathrm{Cr}_{2} \mathrm{O}_{3}$ (second line, 2 wt \%) and $\mathrm{TbCrO}_{3}$ (third line, 7 wt \%); and (b) allowed nuclear reflections for scheelite polymorph (first line), $\mathrm{Cr}_{2} \mathrm{O}_{3}$ (third line), $\mathrm{TbCrO}_{3}$ (fourth line) and allowed magnetic reflections for scheelite polymorph (second line).

obtained from room temperature $\mathrm{x}$-ray diffraction data ${ }^{21}$ were used as starting model for the refinement of the neutron diffraction data collected at $40 \mathrm{~K}$. In the Rietveld analysis all Bragg reflections can be indexed according to the $I 4_{1} / a$ tetragonal space group. However, small amounts of $\mathrm{TbCrO}_{3}$ and $\mathrm{Cr}_{2} \mathrm{O}_{3}$ were observed and included in the refinement. The analysis of the data gives essentially the same results as those obtained from room temperature $\mathrm{x}$-ray diffraction data. $^{21}$ In the $I 4_{1} / a$ space group the $\mathrm{Tb}^{3+}$ and $\mathrm{Cr}^{5+}$ cations occupy the special positions $4 b(0,1 / 4,5 / 8)$ and $4 a(0,1 / 4$, $1 / 8)$, respectively, and the oxygen atoms are located at the general position $16 f(x, y, z)$. Note that the neutron diffraction data show the presence of $\mathrm{Cr}_{2} \mathrm{O}_{3}(2 \mathrm{wt} \%)$ together with $\mathrm{TbCrO}_{3}(7 \mathrm{wt} \%)$. The refined parameters, main bond distances, angles, and agreement factors for the scheelite polymorph of the $\mathrm{TbCrO}_{4}$ oxide at $40 \mathrm{~K}$ are included in Table I.

The crystal structure of the scheelite polymorph is shown in Fig. 2(b). As in the case of the zircon polymorph, Fig. 2(a), $\mathrm{Tb}^{3+}$ and $\mathrm{Cr}^{5+}$ are coordinated by eight and four oxygen atoms forming bisdisphenoids $\left[\mathrm{TbO}_{8}\right]$ and tetrahedra $\left[\mathrm{CrO}_{4}\right]$, respectively. The zircon-type structure can be described as formed by edge sharing chains of bisdisphenoids running parallel to the $a$ axis, which are connected each other by $\left[\mathrm{CrO}_{4}\right]$ tetrahedra units along the $c$ axis. ${ }^{25,26}$ However in the scheelite-type structure those polyhedra form chains of alternate $\left[\mathrm{CrO}_{4}\right]$ and $\left[\mathrm{RO}_{8}\right]$ units sharing corners along the $a$ axis of the structure. Along the $c$ axis the $\left[\mathrm{TbO}_{8}\right]$ polyhedra of the two mentioned chains share edges giving rise to dimeric 
TABLE I. Atomic parameters, main interatomic distances and bond angles for the scheelite polymorph of $\mathrm{TbCrO}_{4}$, calculated from the Rietveld refinement of neutron diffraction data obtained at 2 and $40 \mathrm{~K}$. The corresponding reliability factors are also included.

\begin{tabular}{|c|c|c|c|c|c|}
\hline \multicolumn{6}{|c|}{ Scheelite $\mathrm{TbCrO}_{4}$} \\
\hline \multicolumn{4}{|l|}{ Radiation } & $\mathrm{ND}^{\mathrm{a}} / \mathrm{TOF}^{\mathrm{b}}$ & ND/TOF \\
\hline \multicolumn{4}{|l|}{ Temperature (K) } & 40 & 2 \\
\hline \multicolumn{4}{|l|}{ Space group } & $I 4_{1} / a$ & $I 4_{1} / a$ \\
\hline \multirow[t]{2}{*}{ Cell parameters $(\AA)$} & $a$ & & & $5.03674(10)$ & $5.03038(6)$ \\
\hline & $c$ & & & $11.3734(4)$ & $11.3705(4)$ \\
\hline \multirow[t]{3}{*}{ Rietveld $R$ factors } & $R_{p}$ & & & 0.132 & 0.0962 \\
\hline & $R_{\mathrm{wp}}$ & & & 0.076 & 0.0742 \\
\hline & $\chi^{2}$ & & & 1.67 & 3.24 \\
\hline \multirow[t]{2}{*}{ Bragg $R$-factors } & $R_{B}$ & & & 0.010 & 0.0314 \\
\hline & $R_{F}$ & & & 0.014 & 0.0179 \\
\hline \multirow{5}{*}{$\begin{array}{l}\text { Atoms, Wyckoff positions, occupations, } \\
x / a, y / b, z / c\end{array}$} & $\mathrm{~Tb}$ & $4 b$ & 1 & $0,1 / 4,5 / 8$ & $0,1 / 4,5 / 8$ \\
\hline & $\mathrm{Cr}$ & $4 a$ & 1 & $0,1 / 4,1 / 8$ & $0,1 / 4,1 / 8$ \\
\hline & $\mathrm{O}$ & $16 f$ & 1 & $0.2405(4)$ & 0.2405 \\
\hline & & & & $0.61158(25)$ & 0.61158 \\
\hline & & & & $0.54751(13)$ & 0.54751 \\
\hline \multicolumn{4}{|l|}{$B_{\text {iso }}\left(\AA^{2}\right)$} & $0.024(6)$ & $0.0221(10)$ \\
\hline \multicolumn{5}{|l|}{ Magnetic $R$-factor } & 0.0159 \\
\hline \multicolumn{6}{|c|}{ Main interatomic distances $(\AA)$ and bond angles (deg) } \\
\hline \multirow[t]{2}{*}{$d(\mathrm{Cr}-\mathrm{O})$} & \multirow{2}{*}{\multicolumn{3}{|c|}{$1.7237(18) \times 4$}} & $d(\mathrm{~Tb}-\mathrm{O})$ & $2.3582(18) \times 4$ \\
\hline & & & & & $2.4089(15) \times 4$ \\
\hline \multirow[t]{4}{*}{$\angle(\mathrm{O}-\mathrm{Cr}-\mathrm{O})$} & \multicolumn{3}{|c|}{$118.50(15) \times 2$} & $\angle(\mathrm{O}-\mathrm{Tb}-\mathrm{O})$ & $136.11(13) \times 2$ \\
\hline & \multirow{3}{*}{\multicolumn{3}{|c|}{$105.16(16) \times 4$}} & & $98.03(11) \times 4$ \\
\hline & & & & & $131.56(11) \times 4$ \\
\hline & & & & & $70.17(8) \times 2$ \\
\hline$\angle(\mathrm{Cr}-\mathrm{O}-\mathrm{Tb})$ & \multicolumn{4}{|c|}{$120.71(7) \times 1$} & \\
\hline
\end{tabular}

${ }^{\mathrm{a} N D}=$ neutron diffraction.

${ }^{\mathrm{b}} \mathrm{TOF}=$ time-of-flight.

units, $\left[\mathrm{Tb}_{2} \mathrm{O}_{14}\right]$. In both type structures the $\left[\mathrm{CrO}_{4}\right]$ tetrahedra are well isolated one from another.

From the crystallographic point of view, according to Nyman et al. ${ }^{26}$ the scheelite-type structure can be derived by simultaneous twining of the zircon-type structure in [200], [020], and [002] directions. Experimentally, Kusaba et al. ${ }^{27}$ concluded that the [110] direction in zircon-type structure becomes the [100] direction in the scheelite-type structure, consistent with an electron diffraction study that determined that the [110] direction of the scheelite-type structure is parallel to the [100] of the zircon-type one. ${ }^{28}$ Bond breaking atom diffusion are not considered in this zircon-scheelite phase transition, only a more effective packing in the scheelite form due to the cooperative rotations of $\left[\mathrm{SiO}_{4}\right]$ tetrahedra, justifying the increase of $10 \%$ in density going from zircon to scheelite-form. These results point toward a the displacive-type structural transition. However, recent $a b$ initio calculations show that the zircon to scheelite transition is a first order and reconstructive-type one. ${ }^{29}$ This reconstructive mechanism explains why the scheelite phases are metastable at ambient pressure and do not return to the zircon phase on release of pressure. ${ }^{30,31}$ These results agree with our structural refinements, where it can be observed that the $\mathrm{Cr}-\mathrm{O}$ and $\mathrm{Tb}-\mathrm{O}$ distances do not change very much in both zircon and scheelite polymorphs, but there are remarkable changes in bond angles. These important changes appear to support the reconstructive model to explain this structural zircon-scheelite phase transition. In spite of these theoretical and experimental studies, the microscopic mechanism of this zircon-to-scheelite phase transition still remains an open question. We have observed that the zircon polymorph of $\mathrm{TbCrO}_{4}$ is formed by heating the scheelite polymorph at 673 $\mathrm{K}$ in an oxygen flow.

\section{B. Specific heat measurements}

In a previous work, we have studied the magnetic behavior of scheelite-type $\mathrm{TbCrO}_{4}$ from the magnetic susceptibility temperature dependence and isothermal magnetization curves as a function of the external magnetic field. ${ }^{21}$ Antiferromagnetic ordering, $T_{N}=29 \mathrm{~K}$, in which both $\mathrm{Tb}^{3+}$ and $\mathrm{Cr}^{5+}$ sublattices are involved was proposed. Furthermore, a meta- 
a)

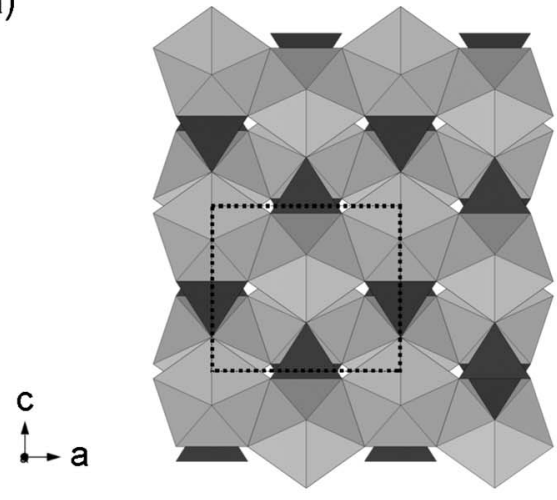

b)

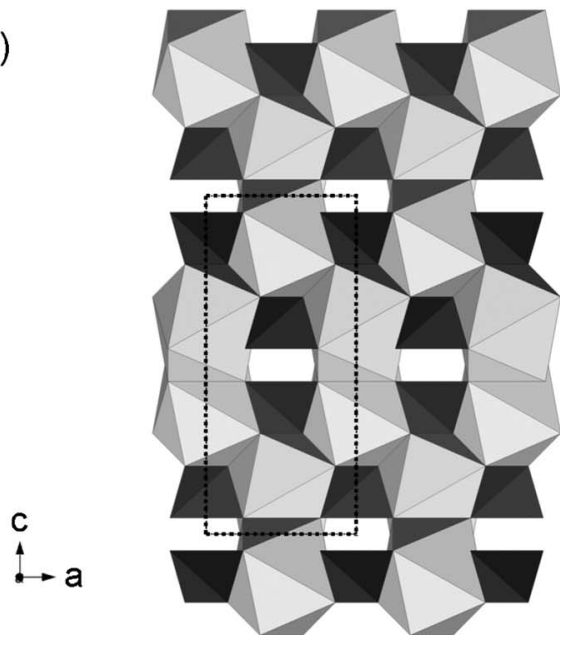

FIG. 2. Perspective view of the $a c$-plane of (a) the zircon-type structure and (b) the scheelite-type structure, showing the arrangement of $\left[\mathrm{TbO}_{8}\right]$ bisdisphenoids polyhedra (light gray) and $\left[\mathrm{CrO}_{4}\right]$ tetrahedra (dark gray).

magnetic transition was observed at $15 \mathrm{~K}$ with a critical magnetic field of $2.6 \mathrm{~T}$. By contrast, the zircon-type $\mathrm{TbCrO}_{4}$ oxide shows the onset of ferromagnetic interactions at $22 \mathrm{~K}$. This behavior is fully confirmed by the determined magnetic structure where the terbium and chromium magnetic sublattices are ferromagnetically coupled in the basal plane of zircon-type structure. ${ }^{3,6}$

The specific heat of scheelite-type $\mathrm{TbCrO}_{4}$ as a function of temperature and magnetic field is shown in Fig. 3. The most striking feature is the sharp $\lambda$ peak at $T_{N}=29 \mathrm{~K}$, which is ascribed, in view of the susceptibility data, ${ }^{21}$ to the threedimensional antiferromagnetic ordering of the magnetic moments of the $\mathrm{Tb}^{3+}$ and $\mathrm{Cr}^{5+}$ ions. The application of an external magnetic field significantly influences the mentioned anomaly. The temperature of the transition decreases gradually with increasing magnetic field and almost disappears at an applied field of $8 \mathrm{~T}$. This field-dependent effect also indicates the magnetic origin of this anomaly. At about $15 \mathrm{~K}$ a small shoulder in the heat capacity is seen. A temperature dependence of the molecular field ${ }^{32,33}$ probably could be the origin of this behavior. Additionally a small contribution coming from the Schottky anomaly due to the splitting of the

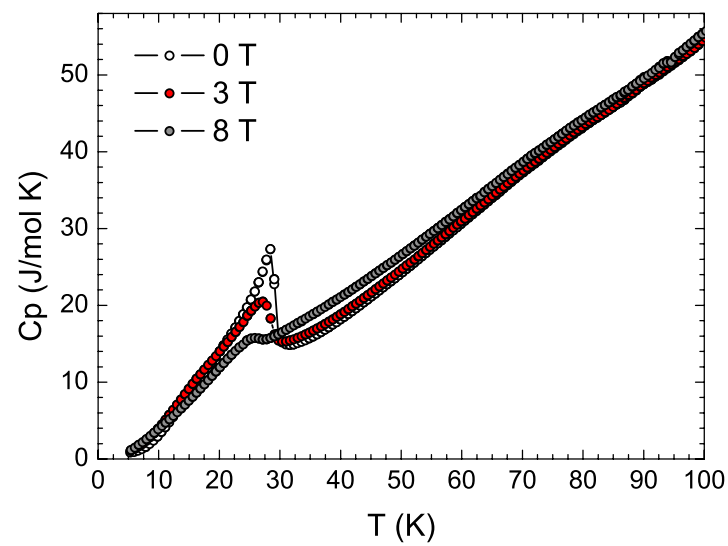

FIG. 3. (Color online) Temperature dependence of the total specific heat measured at different magnetic fields for scheelite-type $\mathrm{TbCrO}_{4}$.

electronic ground state ${ }^{7} F_{6}$ of the $\mathrm{Tb}^{3+}$ ion from the $\mathrm{TbCrO}_{3}$ oxide present in our sample ( $7 \mathrm{wt} \%$ ) will surely also exists in this temperature range.

The calculation of the magnetic contribution to the specific heat for the scheelite-type $\mathrm{TbCrO}_{4}$ oxide requires the subtraction of the lattice contribution. This calculation can be done following different procedures. One way is to use the specific heat data for a diamagnetic compound with the same crystal structure but, until now, such compound has not yet been obtained. A second method is based on the fit of the heat capacity data above $T_{N}$ using a Debye model to reproduce the lattice contribution down to the lowest temperature. However, this data treatment cannot be performed since two unknown heat capacity contributions corresponding to short range magnetic interactions above the magnetic ordering temperature and the Schottky anomaly due to the crystal field splitting of the ${ }^{7} F_{6}$ ground term of $\mathrm{Tb}^{3+}$ are present. Considering these limitations, the lattice contribution was estimated from the specific heat data of the isomorphous $\mathrm{YCrO}_{4}$ oxide recently reported by Long et al. ${ }^{19}$ This oxide shows an antiferromagnetic transition at $T_{N}=18.5 \mathrm{~K}$ in which only the $\mathrm{Cr}^{5+}$ ions are involved. Therefore, as a first approximation, it can be assumed that the data between 40 and $100 \mathrm{~K}$ are close to the lattice contribution for the scheelite-type $\mathrm{YCrO}_{4}$ because, in this case and temperature range, there is no Schottky contribution and the magnetic contribution is small compared with the phononic part. The lattice contribution between 5 and $40 \mathrm{~K}$ has been estimated using a Debye function with a characteristic Debye temperature $\left(\theta_{D}\right)$ of $407 \mathrm{~K}$; this is the effective $\theta_{D}$ for $\mathrm{YCrO}_{4}$ at $40 \mathrm{~K}$. This rough lattice heat capacity for $\mathrm{YCrO}_{4}$ must be scaled to $\mathrm{TbCrO}_{4}$ using a corresponding state law in order to take into account the mass and volume differences between both compounds. However, the Schottky anomaly due to the crystal field splitting of the ${ }^{7} F_{6}$ ground term of $\mathrm{Tb}^{3+}$ cannot be estimated due to the unknown energy level scheme, and unfortunately prevents the determination of the appropriate factor. Therefore, to a first approximation, a direct subtraction has been done in order to obtain the magnetic contribution to the specific heat, Fig. 4. The magnetic entropy after subtracting of the lattice contribution, is $21.7 \mathrm{~J} / \mathrm{mol} \mathrm{K}$ (calculated between 5 and 100 


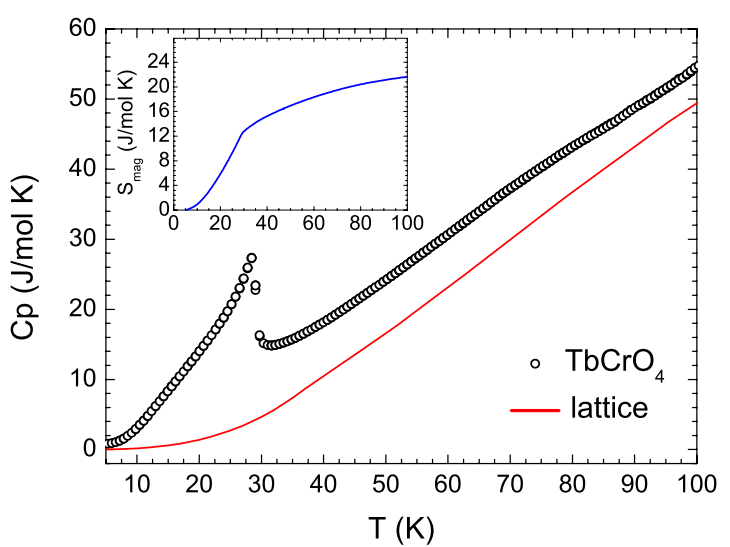

FIG. 4. (Color online) Temperature dependence of the total specific heat measured at $0 \mathrm{~T}$ for scheelite-type $\mathrm{TbCrO}_{4}$ (open circles) and lattice contribution (solid line). The inset shows the variation of the magnetic entropy associated with the magnetic ordering of $\mathrm{Cr}^{5+}$ and $\mathrm{Tb}^{3+}$ for scheelite-type $\mathrm{TbCrO}_{4}$ at $0 \mathrm{~T}$.

$\mathrm{K})$ which is close to the theoretical value of $27.08 \mathrm{~J} / \mathrm{mol} \mathrm{K}$ for $\mathrm{Cr}^{5+}(S=1 / 2)$ and $\mathrm{Tb}^{3+}(J=6)$ as can be observed in the inset in Fig. 4. Of course, a more complete analysis could be done with the knowledge of the energy level scheme which would allow us to evaluate the Schottky anomaly and, after subtracting it from the $\mathrm{TbCrO}_{4}$ heat capacity, to apply the corresponding state law in the high-temperature range (80$100 \mathrm{~K}$ ) well above the antiferromagnetic transition where the short-range magnetic contribution can be considered negligible.

\section{Magnetic structure determination}

The magnetic structure of the scheelite polymorph of $\mathrm{TbCrO}_{4}$ has been determined from the analysis of the neutron diffraction powder pattern collected at $2 \mathrm{~K}$, Fig. 1(b). The refined structure of scheelite-type $\mathrm{TbCrO}_{4}$ at $40 \mathrm{~K}$ (Table I) was used as model for the analysis of the data, taking into account the presence of $\mathrm{TbCrO}_{3}(7 \mathrm{wt} \%)$ and $\mathrm{Cr}_{2} \mathrm{O}_{3}(2 \mathrm{wt} \%)$. Thus, the pattern at $2 \mathrm{~K}$ shows the onset of

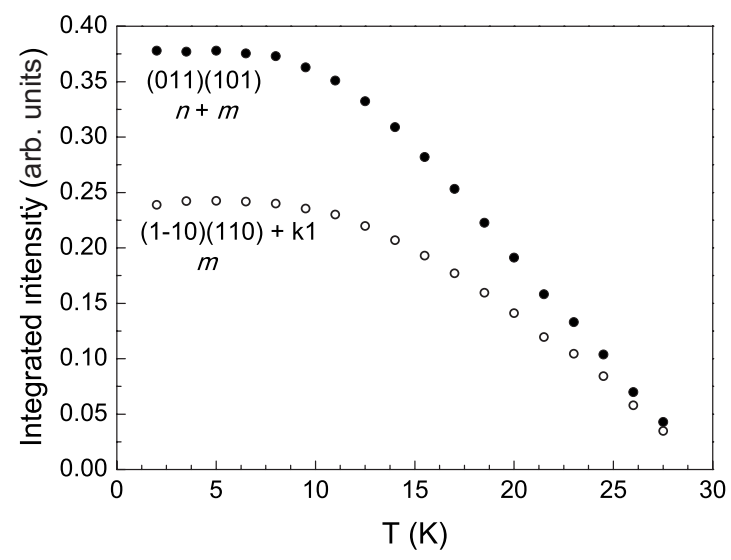

FIG. 5. Integrated intensity of two selected magnetic reflections as a function of temperature.

reflections forbidden in the structural space group, indicative of a decrease in symmetry due to the magnetic ordering, together with a marked increasing of the intensity of few nuclear reflections with regard to the pattern at $40 \mathrm{~K}$ (Fig. 1). These facts can be ascribed to the occurrence of long-range magnetic ordering and the magnetic reflections can only be indexed successfully on the basis of the coincidence between the chemical and magnetic cells, with the propagation vector $\mathbf{k}=(0,0,0)$. The onset of the magnetic ordering was estimated from the thermal variation of the integrated intensity of the magnetic reflections with $d$ spacings of 3.6 and $4.6 \AA$, see Figs. 1(b) and 5. The estimated ordering temperature, $T_{N}=29 \mathrm{~K}$, agrees with that one determined from magnetic susceptibility measurements. ${ }^{21}$ Moreover, the plot indicates that $\mathrm{Cr}^{5+}$ and $\mathrm{Tb}^{3+}$ magnetic sublattices become ordered at the same temperature. The magnetic symmetry analysis was performed with the BASIREPS (Ref. 23) program following the Bertaut ${ }^{34}$ procedure. Tables II and III shows the onedimensional irreducible representations $\Gamma_{\mathrm{i}}(i=1-8)$ of the little group $G_{k}$ and the corresponding basis vectors by each of the irreducible representations. The magnetic representation (Table II) associated with $4 a\left(\mathrm{~Tb}^{3+}\right)$ and $4 b\left(\mathrm{Cr}^{5+}\right)$ sites is the same and it may be reduced to

TABLE II. Irreducible representations of the little group $G_{k}(\mathbf{k}=(0,0,0))$ and magnetic representations associated with the $4 a$ and $4 b$ Wyckoff sites.

\begin{tabular}{lcccccccc}
\hline \hline & \multicolumn{7}{c}{ Symmetry operators $g$} \\
\cline { 2 - 9 }$\Gamma_{v}$ & $\{1 \mid 000\}$ & $\left\{2_{z} \mid \frac{1}{2} 0 \frac{1}{2}\right\}$ & $\left\{4_{z}^{+} \mid \frac{3}{4} \frac{1}{4} \frac{1}{4}\right\}$ & $\left\{4_{z}^{-} \mid \frac{3}{4} \frac{3}{4} \frac{3}{4}\right\}$ & $\{\overline{1} \mid 000\}$ & $\left\{m_{x y} \mid \frac{1}{2} 0 \frac{1}{2}\right\}$ & $\left\{\overline{4}_{z}^{+} \mid \frac{1}{4} \frac{3}{4} \frac{3}{4}\right\}$ & $\left\{\overline{4}_{z}^{-} \mid \frac{1}{4} \frac{1}{4}\right\}$ \\
\hline$\Gamma_{1}$ & 1 & 1 & 1 & 1 & 1 & 1 & 1 & 1 \\
$\Gamma_{2}$ & 1 & 1 & 1 & 1 & -1 & -1 & -1 & -1 \\
$\Gamma_{3}$ & 1 & 1 & -1 & -1 & 1 & 1 & -1 & -1 \\
$\Gamma_{4}$ & 1 & 1 & -1 & -1 & -1 & -1 & 1 & 1 \\
$\Gamma_{5}$ & 1 & -1 & $i$ & $-i$ & 1 & -1 & $i$ & $-i$ \\
$\Gamma_{6}$ & 1 & -1 & $i$ & $-i$ & -1 & 1 & $-i$ & $i$ \\
$\Gamma_{7}$ & 1 & -1 & $-i$ & $i$ & 1 & -1 & $-i$ & $i$ \\
$\Gamma_{8}$ & 1 & -1 & $-i$ & $i$ & -1 & 1 & $i$ & $-i$ \\
$4 a \mathrm{Cr}^{5+} \Gamma_{\text {mag }}$ & 6 & -2 & 0 & 0 & 0 & 0 & 2 & 2 \\
$4 b \mathrm{~Tb}^{3+} \Gamma_{\text {mag }}$ & 6 & -2 & 0 & 0 & 0 & 0 & 2 & 2 \\
\hline \hline
\end{tabular}


TABLE III. Basis vectors of the irreducible representations for $\mathrm{Cr}^{5+}$ and $\mathrm{Tb}^{3+}$ sublattices in the space group $I 4_{1} / a$ with $\mathbf{k}=(0,0,0)$.

\begin{tabular}{|c|c|c|c|c|c|c|c|c|c|c|c|c|}
\hline \multirow[b]{4}{*}{ IR } & \multicolumn{12}{|c|}{ Basis vectors } \\
\hline & \multicolumn{6}{|c|}{$4 a$} & \multicolumn{6}{|c|}{$4 b$} \\
\hline & \multicolumn{3}{|c|}{$\mathrm{Cr}^{5+}(1)$} & \multicolumn{3}{|c|}{$\mathrm{Cr}^{5+}(2)$} & \multicolumn{3}{|c|}{$\mathrm{Tb}^{3+}(1)$} & \multicolumn{3}{|c|}{$\mathrm{Tb}^{3+}(2)$} \\
\hline & $x$ & $y$ & $z$ & $x$ & $y$ & $z$ & $x$ & $y$ & $z$ & $x$ & $y$ & $z$ \\
\hline$\Gamma_{1}$ & 0 & 0 & 1 & 0 & 0 & 1 & 0 & 0 & 1 & 0 & 0 & 1 \\
\hline$\Gamma_{4}$ & 0 & 0 & 1 & 0 & 0 & -1 & 0 & 0 & 1 & 0 & 0 & -1 \\
\hline$\Gamma_{5}$ & 1 & $-i$ & 0 & 1 & $-i$ & 0 & 1 & $-i$ & 0 & 1 & $-i$ & 0 \\
\hline$\Gamma_{6}$ & 1 & $i$ & 0 & -1 & $-i$ & 0 & 1 & $i$ & 0 & -1 & $-i$ & 0 \\
\hline$\Gamma_{7}$ & 1 & $i$ & 0 & 1 & $i$ & 0 & 1 & $i$ & 0 & 1 & $i$ & 0 \\
\hline$\Gamma_{8}$ & 1 & $-i$ & 0 & -1 & $i$ & 0 & 1 & $-i$ & 0 & -1 & $i$ & 0 \\
\hline
\end{tabular}

$$
\Gamma_{\text {mag }}=\Gamma_{1}+\Gamma_{4}+\Gamma_{5}+\Gamma_{6}+\Gamma_{7}+\Gamma_{8} .
$$

If the same irreducible representations (Table III) are involved for both sites only $\Gamma_{4}\left(G_{z}\right)$ permits the exclusively antiferromagnetic coupling of chromium and terbium magnetic moments and therefore the magnetic structure is a collinear one where both magnetic moments are parallel to the $c$ axis. The magnetic structure is defined in the primitive $P 1$ space group and the best agreement between the experimental and calculated neutron diffraction profiles was obtained when $\mathrm{Cr}^{5+}$ and $\mathrm{Tb}^{3+}$ magnetic moments take values of $1.089(9) \mu_{B}$ and $8.97(3) \mu_{B}$, respectively. Therefore, in this magnetic structure the $\mathrm{Tb}^{3+}$ cations are antiferromagnetically coupled inside the $\left[\mathrm{Tb}_{2} \mathrm{O}_{14}\right]$ dimers and these dimers are antiferromagnetically coupled along the $c$ axis. The $\mathrm{Cr}^{5+}$ cations also show antiferromagnetic couplings along the $c$ axis. Finally, both sublattices are antiferromagnetic coupled, see Fig. 6.

In order to understand the mechanism through the observed magnetic interactions take place in both polymorphs, two important facts have to be kept in mind. First, the lack of

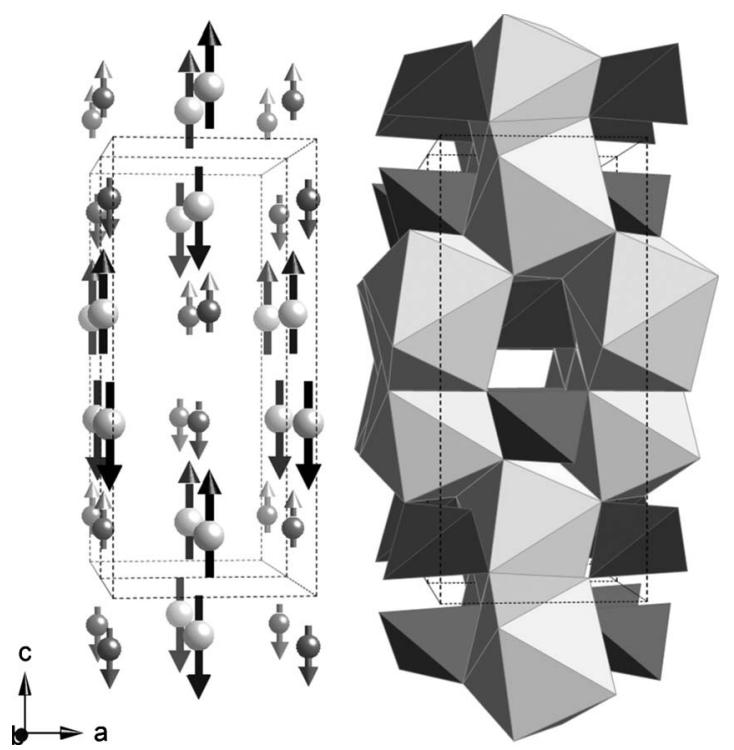

FIG. 6. Proposed magnetic structure for scheelite-type $\mathrm{TbCrO}_{4}$ at $2 \mathrm{~K}$ (left), together with the nuclear structure (right). long-range magnetic ordering down to $61 \mathrm{mK}$ reported for the isomorphous $\mathrm{TbVO}_{4}$-zircon compound $\left(V^{5+}, d^{0}\right)$ reveals the important role that the $\mathrm{Cr}^{5+}$ plays as promoter of the magnetic interactions on the $\mathrm{Tb}^{3+}$ sublattice. ${ }^{35}$ Second, the superexchange $\mathrm{Cr}-\mathrm{O}-\mathrm{O}-\mathrm{Cr}$ is hindered because of the poor orbital overlapping of chromium atoms located at the center of the isolated $\left[\mathrm{CrO}_{4}\right]$ tetrahedra that constitute zircon- and scheelite-type structures, see Fig. 2. Therefore, the likely magnetic interaction mechanism involves the superexchange pathway $\mathrm{Cr}-\mathrm{O}-\mathrm{Tb}$ and its bond distances and bond angles will determine the change of the sign in the exchange integral from ferromagnetic for the zircon-type polymorph to antiferromagnetic for scheelite-type polymorph. The $\mathrm{Cr}-\mathrm{O}$ and $\mathrm{Tb}-\mathrm{O}$ distances of the corresponding polyhedra do not change very much when going from the zircon to the scheelite polymorph, which could explain the near coincidence of the magnetic ordering temperatures of the two polymorphs. However, there is a remarkable change in the $\mathrm{Cr}$ $\mathrm{O}-\mathrm{Tb}$ angles which seems to be the responsible of the different magnetic behavior observed between polymorphs.

\section{CONCLUSIONS}

The scheelite polymorph of $\mathrm{TbCrO}_{4}$ has been prepared from the zircon form of $\mathrm{TbCrO}_{4}$ under $4 \mathrm{GPa}$ at $823 \mathrm{~K}$ for 30 min. This scheelite $\mathrm{TbCrO}_{4}$ phase can be quenched to ambient conditions and its structural and magnetic properties have been studied. Neutron diffraction data have been used to fully characterize the structure of the high-pressure polymorph of the $\mathrm{TbCrO}_{4}$ oxide. The analysis of the lattice parameters and main interatomic distances appears to indicate that this zircon to scheelite transition induced by pressure is a first order reconstructive phase transition, according to Smirnov et al. $^{29}$ Furthermore, bulk magnetic ${ }^{21}$ and specific heat measurements indicate the presence of antiferromagnetism with an estimated Néel temperature of 29 K. By contrast zircon-type $\mathrm{TbCrO}_{4}$ exhibits a ferromagnetic behavior with a Curie temperature of $22 \mathrm{~K}^{3,6}$ The different magnetic behavior of the polymorphs has been attributed to the differences found in the $\mathrm{Cr}-\mathrm{O}-\mathrm{Tb}$ superexchange through, which the magnetic interactions take place. The analysis of the neutron diffraction pattern at low temperature corresponding to scheelite-type $\mathrm{TbCrO}_{4}$ shows that the magnetic moments of 
$\mathrm{Tb}^{3+}$ and $\mathrm{Cr}^{5+}$ are antiferromagnetically aligned along the $c$ axis of the structure.

\section{ACKNOWLEDGMENTS}

The authors are grateful to Spanish MICINN and FEDER for financial support under Research Projects No. MAT2007-
63497 and No. MAT2007-61621, CONSOLIDER-Ingenio 2010 (Grant No. CSD 2007-00010), and ISIS facilities where the neutron experiments were done. We also thank Robert J. Cava, Shuang Jia, Ni Ni, and Martin Bremholm (Department of Chemistry, Princeton University) for the fruitful discussions.
*Present address: Department of Chemistry, Princeton University, Princeton, NJ 08544, USA; ecliment@princeton.edu

${ }^{1}$ G. Buisson, F. Berthaut, and J. Marescal, C. R. Acad. Sci. Paris 259, 411 (1964).

${ }^{2}$ V. H. Schwarz, Z. Anorg. Allg. Chem. 323, 44 (1963).

${ }^{3}$ E. Jiménez Melero, Ph.D. thesis, Universidad Complutense de Madrid, 2005.

${ }^{4}$ C. E. Rice and W. R. Robinson, Acta Crystallogr., Sect. B: Struct. Crystallogr. Cryst. Chem. 32, 2232 (1976).

${ }^{5}$ E. Jiménez Melero, J. Isasi, and R. Sáez Puche, J. Alloys Compd. 323-324, 115 (2001).

${ }^{6}$ G. Buisson, F. Tcheou, F. Sayetal, and K. Schenesman, Solid State Commun. 18, 871 (1976).

${ }^{7}$ H. Walter, G. Kable, K. Mulder, C. Schopper, and N. Schwarz, Int. J. Magn. 5, 129 (1973).

${ }^{8}$ J. A. Hodges, P. Imbert, and G. Jehanno, J. Phys. (France) 43, 1249 (1982).

${ }^{9}$ J. C. Nipko, C. K. Loong, S. Kern, M. M. Abraham, and L. A. Boatner, J. Alloys Compd. 250, 369 (1997).

${ }^{10}$ R. Sáez-Puche, E. Jiménez, J. Isasi, M. T. Fernández-Díaz, and J. L. García Muñoz, J. Solid State Chem. 171, 161 (2003).

${ }^{11}$ E. Jiménez-Melero, P. C. M. Gubbens, M. P. Steenvoorden, S. Sakarya, A. Goosens, P. Dalmas de Réotier, A. Yaouanc, J. Rodríguez-Carvajal, B. Beuneu, J. Isasi, R. Sáez-Puche, U. Zimmerman, and J. L. Martínez, J. Phys.: Condens. Matter 18, 7893 (2006)

${ }^{12}$ V. S. Stubican and R. Roy, Z. Kristallogr. 119S, 90 (1963).

${ }^{13}$ J. P. Bastide, J. Solid State Chem. 71, 115 (1987).

${ }^{14}$ D. Errandonea, J. Pellicer-Porres, F. J. Manjón, A. Segura, Ch. Ferrer-Roca, R. S. Kumar, O. Tschauner, J. López-Solano, P. Rodríguez-Hernández, S. Radescu, A. Mujica, A. Muñoz, and G. Aquilanti, Phys. Rev. B 73, 224103 (2006).

${ }^{15}$ V. Panchal, N. Garg, and S. N. Sharma, J. Phys.: Condens. Matter 18, 3917 (2006).

${ }^{16}$ D. Errandonea and F. J. Manjón, Prog. Mater. Sci. 53, 711 (2008).

${ }^{17}$ F. X. Zhang, M. Lang, R. C. Ewing, J. Lian, Z. W. Wang, J. Hu, and L. A. Boatmer, J. Solid State Chem. 181, 2633 (2008).

${ }^{18}$ W. A. Crichton and A. Grzechnik, Z. Kristallogr. - New Cryst. Struct. 219, 337 (2004).

${ }^{19}$ Y. W. Long, L. X. Yang, Y. Yu, F. Y. Li, R. C. Yu, and C. Q. Jin, Phys. Rev. B 75, 104402 (2007).

${ }^{20}$ E. Climent Pascual, J. Romero de Paz, J. M. Gallardo Amores, and R. Sáez Puche, Solid State Sci. 9, 574 (2007).

${ }^{21}$ E. Climent Pascual, J. M. Gallardo Amores, J. Romero de Paz, N. Taira, and R. Sáez Puche, J. Alloys Compd. 488, 524 (2009).

${ }^{22}$ E. Jiménez Melero, N. H. van Dijk, W. H. Kraan, P. C. M. Gubbens, J. Isasi, and R. Sáez Puche, J. Magn. Magn. Mater. 288, 1 (2005).

${ }^{23}$ J. Rodríguez-Carvajal, Physica B 192, 55 (1993).

${ }^{24}$ E. S. R. Gopal, Specific Heat al Low Temperature (Plenum Press, New York, 1966).

${ }^{25}$ E. Jiménez Melero, J. Isasi, and R. Sáez Puche, J. Alloys Compd. 312, 53 (2000).

${ }^{26}$ H. Nyman, B. Hyde, and S. Anderson, Acta Crystallogr., Sect. B: Struct. Sci. 40, 441 (1984).

${ }^{27}$ K. Kusaba, T. Yagi, H. Kikuchi, and Y. Syomo, J. Phys. Chem. Solids 47, 675 (1986).

${ }^{28}$ H. Leroux, W. W. Reinold, C. Koeberl, U. Hornemann, and J.-C. Doukhan, Earth Planet. Sci. Lett. 169, 291 (1999).

${ }^{29}$ M. B. Smirnov, A. P. Mirgorodsky, V. Yu. Kazimirov, and R. Guinebretière, Phys. Rev. B 78, 094109 (2008).

${ }^{30}$ M. Marqués, M. Flórez, J. M. Recio, L. Gerward, and J. S. Olsen, Phys. Rev. B 74, 014104 (2006).

${ }^{31}$ Y. W. Long, L. X. Yang, Y. Yu, Y. Li, X. Lu, Y. L. Liu, and C. O. Jin, J. Appl. Phys. 103, 093542 (2008).

${ }^{32}$ M. Griffel, R. E. Skochdopole, and F. H. Spedding, Phys. Rev. 93, 657 (1954).

${ }^{33}$ L. D. Jennings, R. M. Stanton, and F. H. Spedding, J. Chem. Phys. 27, 909 (1957).

${ }^{34}$ E. F. Bertaut, Acta Crystallogr., Sect. A: Cryst. Phys., Diffr., Theor. Gen. Crystallogr. 24, 217 (1968).

${ }^{35}$ G. A. Gehring, H. G. Kahle, W. Nägele, A. Simon, and W. Wüchner, Phys. Status Solidi B 74, 297 (1976). 